\title{
PREPARATION AND CHARACTERIZATION OF ALGINATE AND CHITOSAN IPC BASED GEL FORMULATION FOR MUCOSAL APPLICATION
}

\author{
SEDA RENÇBER, ${ }^{*}$ CATALINA NATALIA CHEABURU-YILMAZ, ${ }^{* *}$ FADIME AYDIN KÖSE, ${ }^{* * *}$ \\ SINEM YAPRAK KARAVANA* ${ }^{*}$ and ONUR YILMAZ ${ }^{* * * *}$ \\ "Ege University, Faculty of Pharmacy, Department of Pharmaceutical Technology, \\ 35100, Izmir, Turkey \\ ** "Petru Poni" Institute of Macromolecular Chemistry, 700487, Iasi, Romania \\ ${ }^{* * *}$ Ege University, Faculty of Pharmacy, Department of Biochemistry, 35100, Izmir, Turkey \\ ${ }^{* * * *}$ Ege University, Faculty of Engineering, Leather Engineering Department, \\ 35100, Izmir, Turkey \\ \Corresponding author: C. N. Cheaburu-Yilmaz,duncaty@gmail.com
}

Received February 21, 2019

Interpolymeric complexes (IPCs) have the advantage of combined properties of their constituent polymers. They are easy to prepare under mild conditions and do not require high processing costs. The interpolymeric complexes of alginate, chitosan and Nystatin, as antifungal drug systems, represent an alternative for mucosal illness treatment. The composition of the two polymers is a key parameter in obtaining the desired properties for pharmaceutical formulations. IPCs of sodium alginate (SA) and chitosan (CS) were prepared via solution mixing method with various compositions, followed by coacervation in 2-propanol. The outcome obtained from rheological measurements was that the complex SA/CS 60/40 (v/v \%) fulfilled the requirements of a semi-solid formulation, e.g. pseudoplasticity. Nystatin showed very good compatibility with the polymeric system. The drug loaded gels showed better rheological, textural and mechanical properties (e.g. pseudoelasticity, spreadability, surface adhesion etc.). The final gel formulations showed no cytotoxic effects in the cell culture assay.

Keywords: interpolymeric complex, IPC, alginate, chitosan, gel formulation, Nystatin, cell culture, TPA analysis, rheology

\section{INTRODUCTION}

The increasing need for new biodegradable materials for human health care purposes, especially for drug delivery carriers, has led to a great motivation to develop materials that would satisfy the requirements for such systems. An ideal material to be used in pharmaceutics should associate availability with facile processing and storage requirements, long shelf life, versatility and biocompatibility.

In the last decades, the aim of numerous studies has been the development of gels/hydrogels from natural based materials. ${ }^{1,2}$ Among the variety of biodegradable polymers from renewable resources, chitosan and alginates represent a great alternative to obtain the desired materials with biodegradable and non-toxic characteristics. Both polymers have polysaccha- ride structure, with a difference in their anionic and cationic character due to their functional groups. Chitosan is a cationic biopolymer, comprising $\beta-1,4$ linked glucosamine and $\mathrm{N}$ acetyl-D-glucosamine, while alginate possesses carboxylic groups and an alternating structure of guluronic and manuronic acids, giving an anionic character to the final biopolymer.

By the combination of an anionic polymer with a cationic one, an interpolyelectrolyte complex (IPC) can be formed. ${ }^{3}$ IPCs, throughout the time, have found various applications as membranes for soft tissue engineering, ${ }^{4}$ as hydrogels for tissue regeneration, ${ }^{5}$ hydrogels and microparticles for drug delivery systems. ${ }^{6}$ These biomaterials show different features depending on the polymer concentrations and/or formulation 
conditions. A very desired characteristic, alongside biodegradability and biological properties, is bioadhesion, which is necessary for a good retention on the targeted tissue for medical treatments. Therefore, the preparation conditions and physical-chemical characterization of the IPCs for pharmaceutical applications is crucial to optimize the performance of drugs.

Nystatin (NYS) is a polyene antibiotic, which shows both fungistatic and fungicidal activity against a wide range of pathogenic and nonpathogenic yeasts and yeast-like fungi (Candida, Aspergillus, Histoplasma and Coccidioides). ${ }^{7,8}$ It exerts its antifungal activity by binding to sterols in the fungal cell membrane. As a result of this binding, the membrane is no longer able to function as a selective barrier, and potassium and other cellular constituents are lost. The incidence of disseminated fungal infections has risen over the past decade and Candida is now the fourth most commonly encountered nosocomial bloodstream pathogen. NYS is often used in large doses, which vary from 100,000 units (for oral infections) to 1 million (for intestinal ones), in the case of risk of fungal infections in AIDS patients and patients receiving chemotherapy. ${ }^{7}$ NYS is commonly available in liquid and tablet (lozenge) forms. Thus, extemporaneous compounding represents a unique possibility to formulate NYS preparations in other forms, such as oromucosal/oral gel.

Hydrogels with their three-dimensional, polymeric network have found numerous applications due to their unique properties, such as high water content, softness, flexibility, biocompatibility and ease of preparation..$^{8-10}$ Mucoadhesive hydrogels have been shown to prolong both the retention times on the mucosal area and the drug release..$^{11,12}$

The present paper deals with the preparation and characterization of interpolymeric complexes based on alginate and chitosan that can be used as carrier of Nystatin in mucosal delivery. For this purpose, IPCs of alginate and chitosan were prepared via the solution mixing technique in different ratios, followed by coacervation of the complexes in isopropanol. The IPCs and gel formulations were then characterized in terms of structural analysis, swelling behavior, rheological behavior, textural analysis, drug release performance, cytotoxicity, and the findings were discussed in detail, showing an example for determining a proper drug carrier with respect to the application field.

\section{EXPERIMENTAL}

\section{Materials}

Nystatin (NYS) was donated by Deva Holding A.Ş., Istanbul, Turkey. Medium molecular weight chitosan (CS, Brookfield viscosity $200.000 \mathrm{cps}$, degree of deacetylation of 82) and sodium alginate (SA) were purchased from Sigma-Aldrich (St. Louis, MO, USA). All the other materials were of analytical grade.

Preparation of IPCs of sodium alginate and chitosan with and without drug

Solutions of $2 \mathrm{wt} \%$ sodium alginate in water and chitosan in diluted acetic acid $(0.5 \%)$ were prepared prior to complex formation and allowed to rest overnight. The two biopolymer solutions (SA and CS) were then mixed in ratios of 75/25, 60/40 and 50/50 (v/v \%), respectively. An amount equal to $1 \mathrm{wt} \%$ drug (Nystatin - NYS) against the polymeric matrix was then added into the solutions and the mixtures were shaken for 1 day to ensure good homogenization of the system. The complexes were purified by precipitation/coacervation of the mixtures in 2propanol. The isolated gel-like complexes were washed and freeze-dried. Table 1 summarizes the composition of the samples.

\section{Preparation of the gel formulations}

Gel formulations with and without NYS were prepared by mixing the dried IPCs with double distilled water within a range of concentration between 1 and $5 \mathrm{wt} \%$, giving $100 \mathrm{~mL}$ of dispersion/solution of IPC and water. The solutions were mixed overnight and the obtained semi-solid like gel formulations were freeze-dried to be further tested in solid state.

Table 1

Composition of the formulations

\begin{tabular}{lccc}
\hline $\begin{array}{l}\text { Formulation } \\
\text { code }\end{array}$ & $\begin{array}{c}2 \mathrm{wt} \% \mathrm{SA} \\
\text { solution }(\mathrm{mL})\end{array}$ & $\begin{array}{c}2 \mathrm{wt} \% \mathrm{CS} \\
\text { solution }(\mathrm{mL})\end{array}$ & $\begin{array}{c}\text { NYS } \\
(\mathrm{mg})\end{array}$ \\
\hline F1 & 60 & 40 & - \\
F2 & 50 & 50 & - \\
F1* & 60 & 40 & 20 \\
F2* & 50 & 50 & 20 \\
F3 & 75 & 25 & - \\
F3* & 75 & 25 & 20 \\
\hline
\end{tabular}


Investigation methods

Fourier transform infrared spectroscopy (FT-IR)

The freeze-dried formulations were spectroscopically analysed to prove the structural differences in solid state by FT-IR. The ATR-FT-IR spectra were recorded using a Perkin Elmer Spectrum 100 Spectrometer (USA), through reflexion on a diamond crystal with an angle of 45 degrees, resolution of $4 \mathrm{~cm}^{-1}$ within the range of $650-4000 \mathrm{~cm}^{-1}$.

\section{Swelling behaviour of the polymeric hydrogels}

The swelling capacity of the freeze-dried hydrogels was investigated by direct immersion of the formulations in acidic medium, water and in phosphate buffer saline (PBS, pH 7.4) to simulate physiological conditions. The samples were periodically removed from the solution, gently wiped with a soft tissue to remove surface solution, weighed and then carefully placed back into the vessel as quickly as possible.

The swelling degree (Q) was calculated according to Equation (1):

$Q(\%)=\frac{\left(W_{t}-W_{d}\right)}{W_{d}} \times 100$

where $W_{t}$ is the weight of the swollen samples at time $t$ and $W_{d}$ is the weight of the dry sample.

The following equation was used to determine the kinetics of solvent diffusion into the freeze-dried hydrogels: ${ }^{11}$

$\frac{W_{i}}{W_{e q}}=k * t^{n_{r}}$

where $W_{t}$ and $W_{e q}$ represent the amount of solution absorbed by the matrices at time $t$ and at equilibrium, respectively; $k$ is the swelling rate constant or specific rate characteristic of the system and $n_{r}$ is the power diffusion law exponent, which takes into account the type of solvent transport. Equation 2 applies to the initial stages of swelling (swelling degree less than $60 \%)$

\section{Determination of $\mathrm{pH}$}

To investigate the compatibility of the gel bases for mucosal application, their $\mathrm{pH}$ values were measured by a pH meter (NEL Mod.821) at room temperature.

\section{Rheological studies of the gel formulations}

Rheological analysis of the gel formulations was performed at $25 \pm 0.1{ }^{\circ} \mathrm{C}$ with a Haake Mars Rheometer (Thermo Fisher, Germany). The analysis was performed in the flow mode and in conjunction with parallel steel plate geometry (40 $\mathrm{mm}$ diameter) with a gap of $0.3 \mathrm{~mm}$. The gels were applied onto the lower plate, ensuring that formulation shearing was minimized and allowed to equilibrate for at least $1 \mathrm{~min}$ before the analysis. In continuous shear analysis, the upward and downward flow curves for each formulation were measured over shear rates $\left(0-1000 \mathrm{~s}^{-}\right.$ $1)$.
Oscillatory analysis of each formulation was performed after determining its linear viscoelastic region at $25 \pm 0.1{ }^{\circ} \mathrm{C}$, where stress was directly proportional to strain and the storage modulus remained constant. Frequency sweep analysis was performed over the frequency range of 0.01-5.0 Hz, following the application of a constant stress. The standard gap size was $0.3 \mathrm{~mm}$ for each sample. Storage modulus $\left(G^{\prime}\right)$ and loss modulus $\left(G^{\prime \prime}\right)$ of the formulations were determined as previously described. ${ }^{12-15}$ In each case, the rheological properties of the formulations were replicated three times.

\section{Mechanical properties of gel formulations}

Textural analysis was performed to determine the mechanical properties of the formulations using a software-controlled penetrometer (TA.XTplusC Texture Analyser, Stable Micro Systems Ltd., Guilford, UK) equipped with a $500 \mathrm{~g}$ load cell in the Texture Profile Analysis (TPA) mode. The formulations were transferred into a jacketed glass vial $(20 \mathrm{~mL})$ at $25{ }^{\circ} \mathrm{C}$. An analytical probe was twice compressed into each formulation to a defined depth (10 $\mathrm{mm}$ ) with a rate of $2 \mathrm{~mm} / \mathrm{s}$, allowing a delay period (15 s) between the ends of the first and beginning of the second compression. Mechanical parameters (hardness, compressibility, adhesiveness, cohesiveness and elasticity) were derived and calculated from the resultant force-time curve. Experiments were carried out at least three times. From the resultant force-time plots, several mechanical parameters may be derived. As a result of this study, a force-time curve was obtained (Fig. 1) and mechanical parameters (hardness, compressibility, adhesiveness, cohesiveness and elasticity) were calculated.

Hardness is the force required to attain a given deformation and the altitude of the first peak gives the hardness value. Compressibility is the work required to deform the product during the first compression of the probe and the first area under the curve (AUC) defines compressibility. ${ }^{12-18}$ Adhesiveness is the work necessary to overcome the attractive forces between the surface of the sample and the surface of the probe. The second AUC defines adhesiveness. Cohesiveness is the ratio of the area under the force-time curve produced in the second compression cycle to that in the first compression cycle where successive compressions are separated by a defined recovery period. Elasticity is the rate at which the deformed sample returns to its undeformed condition after the removal of the deforming force. Cohesiveness and elasticity values were calculated according to the following equations:

Cohesiveness $=\frac{A U C 3}{A U C 1}$

Elasticity = Time difference $3-4 /$ Time difference $1-2(4)$ 


\section{$U V$-VIS spectrophotometric determination of Nystatin}

The quantitative analysis of the materials based on Nystatin is hindered generally by the limited solubility of Nystatin, which is practically insoluble in water, very slowly soluble in alcohol and chloroform, and soluble in other solvents. Spectrophotochemical methods in ultra violet region seem to be proper for quantification of Nystatin, being simple, fast and sensitive enough. ${ }^{19}$

The UV-VIS measurements could be performed at $\lambda_{\max }$ of $320 \mathrm{~nm}$ wavelength, a region also selected by Rodino et al. $^{19}$ due the advantage that the major ingredients (without chromophores) can be neglected at this wavelength.

The concentration of NYS was determined by using a calibration curve of NYS from a series of standard solutions within a concentration range of $10^{-1}$. $10^{-5} \mathrm{wt} \%$ in ethanol. The dried samples of loaded IPC (approximately $0.2 \mathrm{~g}$ ) were allowed to extract in ethanol for $24 \mathrm{~h}$ under continuous stirring. The UVVIS absorption spectra of standard and IPC samples' solutions were recorded by using a Shimatzu UV1800 Spectrophotometer, using quartz cuvettes with optical path of $5 \mathrm{~mm}$. The spectral range was 260-400 nm. The results obtained are summarized in Table 2 and the calibration curve of Nystatin is presented in Figure 3.

\section{Cell culture studies}

The cytotoxicity potential of the formulations was evaluated by MTT (3-[4,5-dimethylthiazol-2-yl]-2,5diphenyltetrazolium bromide) assay. MTT reagent is a yellow substrate, which produces a dark blue formazan product when incubated with viable cells. The level of the reduction of MTT to form formazan can reflect the level of cell metabolism. A human kidney proximal tubular epithelial cell line (HK-2, ATCC) was cultured in $10 \%$ fetal bovine serum (FBS) supplemented DMEM (Dulbecco's Modified Eagle's medium, Gibco, Thermo Fisher Scientific): F12 medium (Gibco, Thermo Fisher Scientific). A mouse embryo fibroblast cell line (NIH-3T3) was grown in $10 \%$ FBS supplemented DMEM. The cells were maintained at 37 ${ }^{\circ} \mathrm{C}$ in a humidified atmosphere of $5 \% \mathrm{CO}_{2}$ and $95 \%$ air. The cells were plated in 96-well plates, with a density of $5 \times 10^{3}$ cells/well, and incubated at $37{ }^{\circ} \mathrm{C}$ in a humidified atmosphere containing $5 \% \mathrm{CO}_{2}$ for $24 \mathrm{~h}$ for cell attachment. After these preparations, the cells were treated for $48 \mathrm{~h}$ with a 1:1000 dilution of the tested formulation. The cells treated only with medium were considered as control or reference samples. After removing the medium, the cells were washed with phosphate buffer saline (PBS). Approximately $100 \mu \mathrm{L}$ of medium and MTT solution (Life Science, Invitrogen) $(5 \mathrm{mg} / \mathrm{mL})$ were added to each well to determine the cell viability. After incubation for another $4 \mathrm{~h}$, blue-colored formazan crystals were dissolved in dimethyl sulfoxide. The absorbance of the formazan solution was measured in a plate reader (VersaMax) at $540 \mathrm{~nm}$. The ratio of the absorbance of treated samples versus the absorbance of reference or control samples (taken as $100 \%$ ) was expressed as \% cell viability. Cell survival was expressed as the percentage of formazan absorbance. The results were expressed as the mean \pm standard error mean (Mean \pm SEM) from at least three different experiments in triplicate.

\section{Statistical analysis}

Statistical analysis was conducted by ANOVA software, followed by Tukey's test for comparisons between groups. 0.05 was taken as a $\mathrm{P}$ value, to indicate statistical significance $(\mathrm{P}<0.05)$.

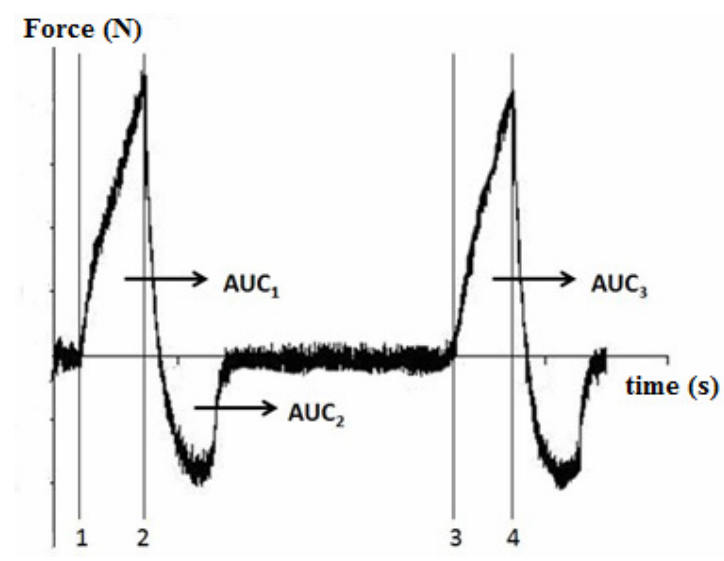

Figure 1: Force-time curve obtained from textural analysis (reproduced from S. Rencber et al. ${ }^{14}$ ) 


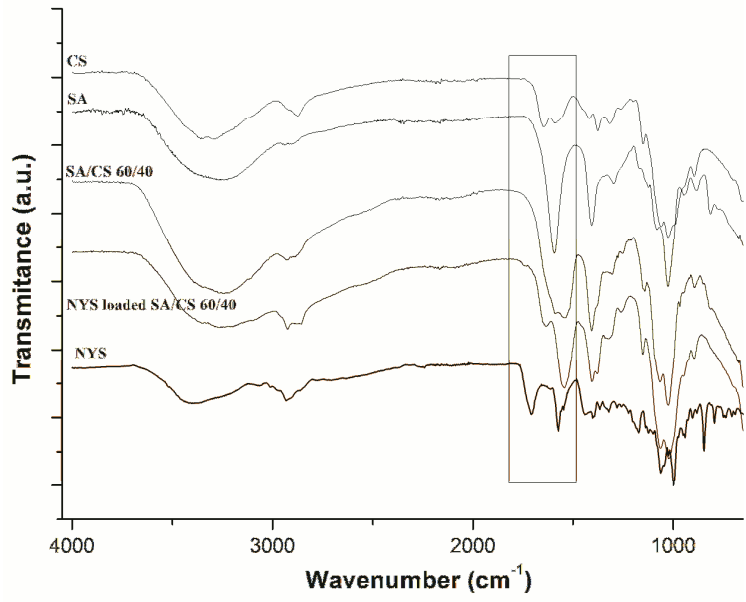

Figure 2: FT-IR spectra of NYS loaded and unloaded complex of alginate and chitosan

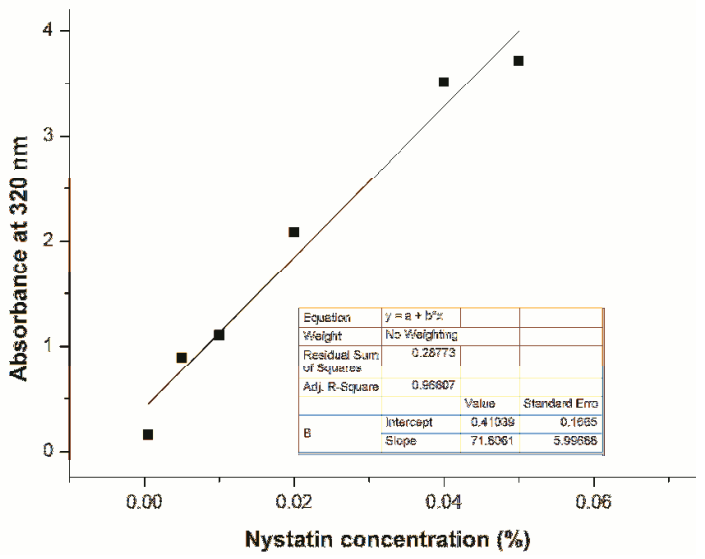

Figure 3: Calibration curve of Nystatin

Table 2

Nystatin concentration determined from UV measurements

\begin{tabular}{lcc}
\hline IPCs code & SA/CS ratio $(\%)$ & NYS $(\mu \mathrm{g} / \mathrm{mL})$ \\
\hline F1* & $60 / 40$ & 2.9 \\
$\mathrm{~F}^{*} *$ & $50 / 50$ & 1.7 \\
\hline
\end{tabular}

\section{RESULTS AND DISCUSSION}

The structure of the prepared IPCs and the presence of Nystatin within polymeric matrices were investigated via FT-IR and UV spectroscopy. The IR spectra of SA, CS, NYS and the ICPs loaded and without drug are given in Figure 2. As it can be noted in the presented spectra, the characteristic peaks of the pure constituents, alginate and chitosan, are both present in the spectrum of IPC. Particularly, the characteristic peaks of chitosan were observed at $2921-2887 \mathrm{~cm}^{-1}\left(-\mathrm{CH}_{3},-\mathrm{CH}_{2}\right), 1636 \mathrm{~cm}^{-1}(\mathrm{C}=\mathrm{O}$ stretch vibration), $1548 \mathrm{~cm}^{-1}$ (secondary amide), $1655 \mathrm{~cm}^{-1}$ corresponding to the amidic band I; $1068-1020 \mathrm{~cm}^{-1}$ (C-O stretching of saccharide moiety). Alginate possessing carboxylate groups showed a characteristic vibration band at 1593 $\mathrm{cm}^{-1}$ and $1408 \mathrm{~cm}^{-1}$, characteristic of C-Ovibration bands. The presence of the characteristic vibration bands of alginate within the spectra of the complex confirmed the formation of an interpolymeric complex; moreover the shifting bands to lower values indicated the formation of hydrogen based bonds between the two polymers.

The presence of the active substance, i.e. NYS, trapped within the macrochains was evidenced by comparing the spectra of the pure drug and the loaded polymeric matrix. NYS as an active substance is a polyene macrolide with a deoxysugar D-mycosamine, an aminoglycoside. Its structure is very complex, as it contains many $\mathrm{OH}$ groups, unsaturated $\mathrm{CH}$ and a piranose cycle. As presented in Figure 2, the main characteristic vibration bands were found within the range $3300-3500 \mathrm{~cm}^{-1}$, as being assigned to $\mathrm{NH}_{2}$ and $\mathrm{OH}$ stretching; $\mathrm{C}=\mathrm{O}$ was observed at $1712 \mathrm{~cm}^{-1}$; $\mathrm{C}-\mathrm{O}$ groups at $1560 \mathrm{~cm}^{-1}$. Although the loading ratio of NYS was low with respect to the polymer matrix, a small peak was observed around 1730 $\mathrm{cm}^{-1}$ in the spectrum of the drug loaded IPC, possibly due to the characteristic $-\mathrm{C}=\mathrm{O}$ absorption of NYS.

The efficiency of drug loading was also examined via UV-VIS spectrophotometry. The amount of NYS released from the polymer matrix after $24 \mathrm{~h}$ of extraction was determined by measuring the absorbance at $320 \mathrm{~nm}$ and using the calibration curve of NYS - Figure 3. The results obtained from the measurements were summarized in Table 2.

In comparison with the theoretical drug loading equal to $20 \mu \mathrm{g} / \mathrm{mL}$, the amount of extracted drug was calculated as 1.7 and $3 \mu \mathrm{g} / \mathrm{mL}$, which corresponds to a ratio of 8.5 and $15 \%$. However, this result can be caused by the low loading ratio or low solubility of the drug in ethanol, and needs further investigation. On the 
other hand, the amount of the extracted drug seemed to be higher for the IPC with 60/40 SA/CS composition, indicating its higher drug loading capacity as a matrix.

The overall results obtained from FT-IR and UV-VIS spectrophotometry nonetheless confirmed both the formation of the interpolymeric complex between the two biopolymers and the presence of the drug within the polymeric complex.

\section{Rheological studies}

The evaluation of the rheological properties of the gel formulations is important to predict in vivo behaviour. ${ }^{14,15}$ Additionally, it provides fundamental information for the assessment of the final product's properties, such as quality, storage stability, effect of formulation variables on product characteristics. $^{20}$ The pseudoplastic or socalled shear thinning behavior is a "must-have" for an easy application of the hydrogel formulation from a tube or syringe to the target site. Additionally, an ideal gel formulation should possess proper gel strength in order to remain on the applied site for a period of time (i.e. $1 \mathrm{~h}$ ).

All the polymeric matrices with different compositions were subjected to rheological measurement to verify their flow behaviour, prior to the preparation of the gel formulations. Among IPCs, the composition of SA/CS of 60/40 and $50 / 50$ indicated a responsive and proper result, while 75/25 exhibited irrational signals to be plotted. Therefore, only the compositions that gave relevant results were used further for gel formulations. Similarly, rheological pre-studies were conducted to choose the proper concentration of polymeric carrier within the gel formulations. By performing the rheological measurements, it was determined that a concentration of $1.5 \%$ of IPC was the best to prepare gel formulations with the desired properties.

The flow behaviour of the polymeric solutions is presented in Figure 4. As observed, the pure alginate solution showed a typical Newtonian fluid behaviour, while the chitosan solution showed very clear gel like properties. Previously, it was determined that the higher the amount of chitosan, the higher strength of the gel. ${ }^{21-24}$ By increasing the amount of chitosan, particularly in the case of IPC with SA/CS 50/50 (v/v \%), the expected flow behavior should be a combined shear thinning with a dilatant specific trend-line; instead, no clear dependence was obtained because of the formed compact structure, the free move of macromolecules being limited. The matrix containing $40 \%$ chitosan (SA/CS 60/40 (v/v \%)) was considered suitable, as the desired pseudoplastic flow behaviour was obtained (Fig. 4).

The viscoelastic characteristics confirmed the outcome of the flow behaviour tests. The higher the amount of chitosan, the higher the strength of the resulting gel formulation was. Figure 5 presents the viscoelastic behaviour of the gel formulations SA/CS 60/40, SA/CS 50/50 and NYS loaded SA/CS 60/40.

The viscoelastic behaviour was studied within the linear viscoelasticity range and dynamic moduli, storage modulus $\left(G^{\prime}\right)$ and loss modulus $\left(G^{\prime \prime}\right)$ were determined.

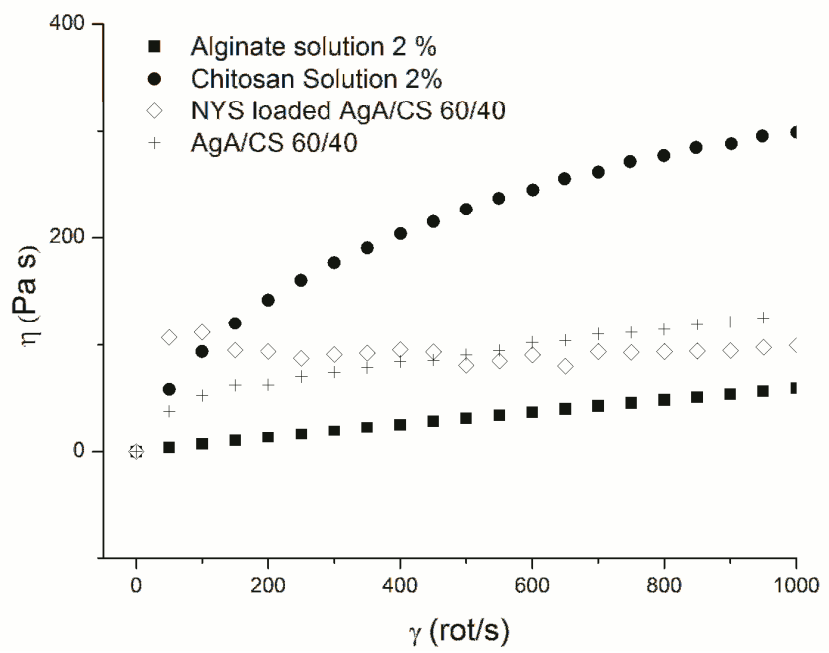

Figure 4: Flow curves of pure polymeric solutions and IPCs, loaded and unloaded with Nystatin, at $25{ }^{\circ} \mathrm{C}$ 


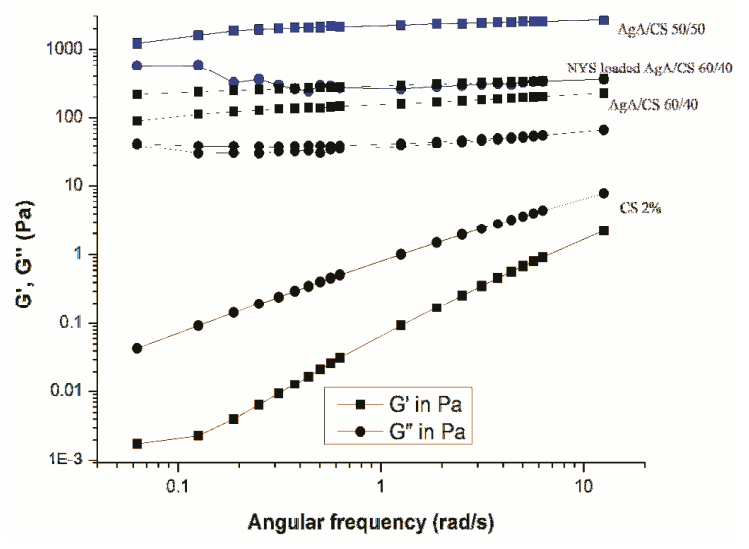

Figure 5: Angular frequency-dependent changes of viscoelastic properties of the formulations

$G^{\prime}$ indicates the elastic modulus and can be correlated with the stored energy necessary to pull back the gels under a shear stress. $G^{\prime \prime}$ illustrates the dissipation of energy when shear stress is applied to the gel. ${ }^{17,24}$

The linear viscoelasticity range was tested using the stress sweep test, which revealed the range of shear stress in which the polymer network would remain intact and interconnected. Beyond the linear viscoelasticity range, the network is expected to entangle and break down, causing gel elasticity to reduce, and as a result, the gel will not be in a stable network as it should. ${ }^{24-27}$

In polymer solutions, at a sufficiently high concentration, there are entanglements among the polymer chains, but there is sufficient time for the polymer chains to disentangle and flow during a single oscillation at low frequencies $\left(G^{\prime \prime}>G^{\prime}\right) .^{25-27}$ Conversely, as the elastic properties of the sample increase, interchain entanglements do not have sufficient time to come apart within the period of single oscillation and $G^{\prime}$ becomes higher than $G^{\prime \prime}$. A gel should exhibit a solid-like mechanical spectrum, that is, $G^{\prime}>G^{\prime \prime}$ throughout the experimentally accessible frequency range, and there should be little frequency dependence of the moduli.

As observed in Figure 5, all the studied gel formulations showed that $G^{\prime}$ is higher that $G^{\prime \prime}$, indicating the gel character. The trend-line of curves $G^{\prime}$ and $G^{\prime \prime}$ as a function of frequency was similar and the strong gel character was evidenced by the parallel distribution of the curves of the two moduli over whole frequency range. At very low angular frequency, there was a tendency of a

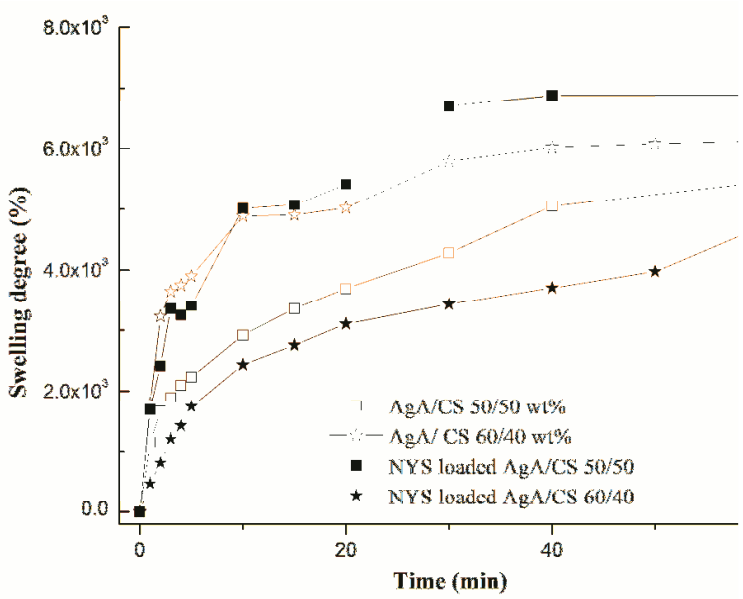

Figure 6: Swelling profiles of the matrix based IPC with different compositions, unloaded and loaded with NYS

crossover point, when $G^{\prime}$ would become equal with $G^{\prime \prime}$ and indicating a sol-gel transition and a structural change.

According to the obtained outcome, the best gel formulation is the one with the composition of SA/CS 60/40 (v/v \%). By loading NYS within the polymeric matrix, the storage modulus of the gel formulation incorporating the drug increased 1.5 times, indicating increased strength (Fig. 5).

The value of phase angle $\left(\tan \delta=G^{\prime \prime} / G^{\prime}\right)$, a measure of the relative contribution of the viscous components to the mechanical properties of the materials, took lower values than unity, confirming the solid gel response (not shown herein). ${ }^{26}$

\section{Swelling ability}

The freeze-dried hydrogels were analysed with regard to their swelling ability by varying the composition of the IPC and by loading NYS within the matrix. Additionally, the swelling capacity in media with different $\mathrm{pH}$ (i.e. $\mathrm{pH}$ acid, water and PBS) was also studied.

As presented in Figure 6, the swelling profiles of two formulations showed maximum swelling ability in water within $1 \mathrm{~h}$ of immersion into water or solvent. The two formulations with different compositions exhibited swelling profiles slightly different when loaded with the active substance. The IPC with the composition SA/CS $50 / 50(\mathrm{v} / \mathrm{v} \%)$ reached the swelling equilibrium after $1 \mathrm{~h}$, having a maximum swelling degree of $5400 \%$ by comparison with the IPC with less chitosan (SA/CS 60/40), whose swelling degree was $6010 \%$. This showed that an increased amount of chitosan led to a denser network, 
hindering the swelling of the IPC matrix. ${ }^{21-24}$ As shown in Figure 6, NYS seemed to play a plastifying role, as the swelling ability of the loaded matrices is different.

The matrix with a higher amount of chitosan (IPC SA/CS 50/50) showed higher ability to intake water within the polymeric macrochains, while the matrix IPC SA/CS 60/40 presented a lower swelling ability. By loading NYS within the polymeric matrices, the maximum swelling ability of the matrix SA/CS $60 / 40$ was $4700 \%$, while the swelling capacity of SA/CS 50/50 was $6800 \%$. This behaviour could be explained by the fact that the matrix with a higher amount of chitosan already had a dense structure and NYS could not be uniformly loaded and was loaded in a smaller amount, as it was also determined by UV-VIS spectrometry. In the case of matrix SA/CS 60/40 (v/v \%), NYS played the typical behaviour of a filler, increasing the structural density; this explaining the lower values of the swelling degree.

The swelling kinetic parameters, e.g. $n_{s w}, k_{s w}$ and $R^{2}$ were determined by applying Equation 2 . The obtained values were summarized in Table 3. According to the swelling kinetic parameters summarized in Table 3, the swelling mechanism of the studied hydrogels is non-Fickian, which indicates that the rate of water molecules diffusion into the network is higher than the rate of polymeric chain relaxation. Parameter $n$, close to 0.5 for NYS loaded SA/CS $60 / 40$ indicated Fickian diffusion, while the matrices SA/CS 50/50 unloaded and loaded with NYS described a relaxation-controlled transport of water molecules.

\section{Determination of $\mathrm{pH}$}

Surface $\mathrm{pH}$ evaluation of mucosal dosage forms is an important aspect for characterization, since an acidic or alkaline $\mathrm{pH}$ may cause irritation to the mucosa. It was therefore necessary to determine if any extreme surface $\mathrm{pH}$ changes occurred with the mucosal bioadhesive gel during the drug release period investigated. ${ }^{14,20}$ The $\mathrm{pH}$ values of all the prepared gel formulations were found within physiological limits (neutral, approximately 6) and they were deemed to be suitable for mucosal administration (Table 3).

\section{Mechanical properties of gel formulations}

Gel formulations should have acceptable mechanical properties, such as high adhesiveness, ease of application to the surface, low hardness, and good retention at the application site, to provide a maximum benefit of the formulation. TPA permits evaluating the textural properties of different semi-solid formulations in order to gather information about the physical gel structure. The parameters derived from this technique (hardness, compressibility, elasticity and cohesiveness) have been proven to be relevant to the performance of local formulations, e.g. ease of removal from the container, ease of application to the surface and retention of the product at the site of application. ${ }^{14,15,17}$ Therefore, the formulations were evaluated according to their mechanical properties. The mechanical properties of the formulations are presented in Table 4.

The hardness and compressibility values of gel formulations should be low in order to take the formulation easily from the container and apply to the mucosal area. ${ }^{14,15,17}$ The results showed that formulation $\mathrm{F} 2 *$ had both a lower hardness value $(46.497 \pm 0.455 \mathrm{mN})$ and a lower compressibility value $(61.797 \pm 2.006 \mathrm{mN} . \mathrm{mm})$, compared to formulation $\mathrm{F}^{*}$. Significant differences were observed with regard to the hardness and compressibility values $(\mathrm{p}<0.05)$. Product elasticity represents the rate at which the deformed sample returns to its undeformed condition. Lower numerical values given by TPA in the elasticity mode indicate greater product elasticity. It was observed that gel formulation F2* had the lowest elasticity value. Adhesiveness, a property related to mucoadhesion, is defined as the work required to detach the probe from the sample, in which its cohesive bonds were broken and describes the relative properties of each candidate formulation. ${ }^{13}$ Formulation $\mathrm{F}^{*}$ had a higher adhesiveness value than formulation $\mathrm{F} 2$ * $(p<0.05)$. Cohesiveness can be described by the effects of repeated shearing stress on the structural properties of formulations. The high value of cohesiveness can be explained by a full structural recovery following gel application. No significant differences were observed between $\mathrm{F} 1^{*}$ and $\mathrm{F} 2 *(\mathrm{p} \geq 0.05)$.

Based on the results of the mechanical properties, all the gels appeared to offer suitable mechanical properties for mucosal application, being in accordance with the results obtained by other researchers. ${ }^{28,29}$ As can be seen from Table 3 , the presence of NYS affected the mechanical properties of the formulations. 
Table 3

Kinetic parameters for alginate based matrices

\begin{tabular}{lcccc}
\hline \multirow{2}{*}{$\begin{array}{l}\text { System } \\
(\mathrm{v} / \mathrm{\%} \%)\end{array}$} & Swelling degree & \multicolumn{3}{c}{ Kinetic parameters } \\
\cline { 3 - 5 } & $(\%)$ & $\mathrm{n}$ & $\mathrm{k}\left(\mathrm{min}^{-\mathrm{n}}\right)$ & $\mathrm{R}^{2}$ \\
\hline SA/CS 50/50 & 5400 & 0.35 & 0.07 & 0.98 \\
SA/CS 50/50+NYS & 6800 & 0.31 & 0.04 & 0.98 \\
SA/CS 60/40 & 6010 & 0.27 & 0.04 & 0.97 \\
SA/CS 60/40+NYS & 4700 & 0.44 & 0.12 & 0.98 \\
\hline
\end{tabular}

Table 4

$\mathrm{pH}$ and mechanical properties of formulations

\begin{tabular}{lcccccc}
\hline Code & $\mathrm{H}(\mathrm{mN}) \pm \mathrm{SD}$ & $\mathrm{C}(\mathrm{mN} . \mathrm{mm}) \pm \mathrm{SD}$ & $\mathrm{A} \pm \mathrm{SD}$ & $\mathrm{E} \pm \mathrm{SD}$ & $\mathrm{Ch} \pm \mathrm{SD}$ & $\mathrm{pH} \pm \mathrm{SD}$ \\
\hline F1 & $41.96 \pm 1.88$ & $86.87 \pm 2.74$ & $64.11 \pm 2.12$ & $1.19 \pm 0.02$ & $1.13 \pm 0.03$ & $6.14 \pm 0.1$ \\
F1 $*$ & $54.61 \pm 0.57$ & $120.75 \pm 0.47$ & $66.51 \pm 1.17$ & $1.02 \pm 0.02$ & $1.04 \pm 0.00$ & $6.16 \pm 0.1$ \\
F2 & $20.63 \pm 2.61$ & $35.56 \pm 5.64$ & $23.07 \pm 1.61$ & $0.99 \pm 0.01$ & $1.06 \pm 0.12$ & $6.01 \pm 0.1$ \\
F2 $*$ & $46.50 \pm 0.45$ & $61.80 \pm 2.01$ & $36.55 \pm 2.01$ & $0.90 \pm 0.01$ & $1.04 \pm 0.04$ & $6.05 \pm 0.1$ \\
\hline
\end{tabular}

H: Hardness, C: Compressibility, A: Adhesiveness, E: Elasticity, Ch: Cohesiveness

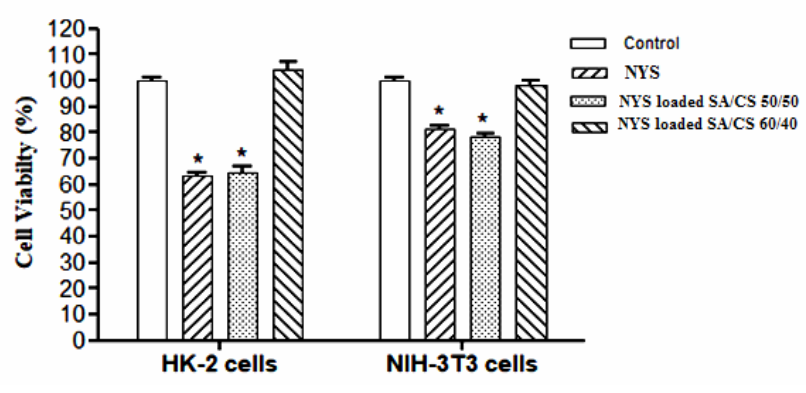

Figure 7: Effects of Nystatin (NYS) and formulations on cell viability 


\section{Cell culture studies}

Cytotoxicity is a key factor when choosing a suitable drug delivery carrier. Figure 7 presents the MTT assays of NYS and NYS-loaded SA/CS 50/50 and NYS loaded 60/40 SA/CS formulations on HK-2 and NIH-3T3 cell lines. It was determined that NYS and NYS loaded SA/CS $50 / 50$ formulations caused a significant decrease in cell viability of HK-2 and NIH-3T3 cells, compared to the control cells $(\mathrm{p}<0.05)$. On the other hand, no cytotoxic effect of the NYS loaded SA/CS 60/40 formulation was found neither towards HK-2 cells nor towards NIH-3T3 cells (p $>0.05$ ). The obtained results could be explained by the fact that SA/CS 60/40 could have the potential to be used as a carrier for Nystatin. The obtained results are supported by other studies. ${ }^{30-}$

The graphs from Figure 7 present a comparison of \% cell viability among control, NYS and NYS-loaded formulations. Untreated cells were used as control. The data were presented as Mean \pm SEM. $(\mathrm{n}=3)$.

\section{CONCLUSION}

Interpolymeric complexes (IPCs) between two natural, biocompatible and non-toxic polymers, alginate and chitosan, were obtained. It was established that, depending on the composition of the complex, the exhibited properties are different with respect to the rheological characteristics and swelling ability. The higher the amount of chitosan within the IPC, the higher the gel strength is. As regards the requirements for pharmaceutical formulations, the composition of SA/CS of $60 / 40(\mathrm{v} / \mathrm{v} \%)$ was determined to be relevant for mucosal application of NYS.

The results obtained from swelling tests were confirmed by the rheological and texture profile measurements, indicating a very good adhesion on the surface of the gel formulations of IPC SA/CS.

Cytotoxicity tests indicated a comparable cell viability of the gel formulations of SA/CS with the reference samples. The drug loading capacity was determined to be around $15 \%$, being moderate, but sufficient for an efficient drug delivery onto the skin surface.

ACKNOWLEDGEMENTS: Cheaburu-Yilmaz C.N. acknowledges the financial support from "The Scientific and Technological Research Council of Turkey (TUBITAK), CoFunded by Marie Curie Actions under FP7", project number
115C078. Additionally, the authors acknowledge Ege University, Faculty of Pharmacy, Pharmaceutical Sciences Research Centre (FABAL) for some instrumental analysis.

Author contributions: C.N.C.Y. synthesized samples, performed analyses and wrote the paper; S.R. conducted some analysis, contributed to rheological and pharmaceutical approaches and partially wrote the paper; F.A.K. conducted cytotoxicity studies and interpreted the data, O.Y. performed some physical chemical characterization, edited and reviewed the manuscript; S.Y.K. performed some characterization and contributed to rheological and pharmaceutical approaches.

\section{REFERENCES}

1 F. Machado Croisfelt, L. Lacalendola Tundisi, J. Artem Ataide, E. Silveira, E. Basile Tambourgi et al., J. Mater. Sci., 54, $10963 \quad$ (2019), https://doi.org/10.1007/s10853-019-03557-x

2 T. Zhu, J. Mao, Y. Cheng, H. Liu, L. Lv et al., Adv. Mater. Interfaces, $1900761 \quad$ (2019), https://doi.org/10.1002/admi.201900761

3 C. N. Cheaburu and G. G. Bumbu, in "Environmentally Degradable Materials Based on Multicomponent Polymeric Systems", edited by C. Vasile, CRC Press Taylor and Francis Group, 2009, pp. 427-498,

https://www.crcpress.com/Environmentally-

Degradable-Materials-based-on-MulticomponentPolymeric/Vasile/p/book/9789004164109

4 K. Wang and Z. He, Int. J. Pharm., 244, 117 (2002), https://doi.org/10.1016/S0378-5173(02)003241

P. G. Park, W. S. W. Shalaby and H. Park, "Biodegradable Hydrogels for Drug Delivery", $1^{\text {st }}$ ed., CRC Press, Lancaster, PA, 1993, https://www.crcpress.com/Biodegradable-Hydrogelsfor-Drug-Delivery/Park-ParkShalaby/p/book/9781566760041

6 C. Vasile, R. P. Dumitriu, C. N. Cheaburu and A. M. Oprea, Appl. Surf. Sci., 256, 65 (2009), https://doi.org/10.1016/j.apsusc.2009.04.120

7 Z. Sklenar, V. Scigel, K. Horackova and O. Slanar, Acta Poloniae Pharm. Drug Res., 70, 759 (2013), https://europepmc.org/abstract/med/23923400

8 C. S. Satish, K. P. Satish and H. G. Shivakumar, Indian J. Pharm. Sci., 68, 133 (2006), https://doi.org/10.4103/0250-474X.25706

9 E. Calo and V. V. Khutoryanskiy, Eur. Polym. J., 65, 252 (2015), https://doi.org/10.1016/j.eurpolymj.2014.11.024

10 S. T. Tanriverdi, C. N. Cheaburu-Yilmaz, S. Carbone and O. Ozer, Pharm. Dev. Technol., 23, 815 
(2018),

https://doi.org/10.1080/10837450.2016.1268158

11 I. G. Needleman, F. C. Smales and G. P. J. Martin, Clin. Periodontol., 24, $394 \quad$ (1997), https://doi.org/10.1111/j.1600-051x.1997.tb00203.x

12 S. Senel, G. Ikinci, S. Kas, A. Yousefi-Rad, M. F. Sargon et al., Int. J. Pharm., 193, 197 (2000), https://doi.org/10.1016/S0378-5173(99)00334-8

13 R. W. Korsmeyer, S. R. Lustig and N. A. Peppas, J. Polym. Sci. B: Polym. Phys., 24, 395 (1986), https://doi.org/10.1002/polb.1986.090240214

14 S. Rencber, S. Y. Karavana, Z. Ay Şenyigit, B. Erac, M. Hoşgor Limoncu et al., Pharm. Dev. Technol., $\quad 22, \quad 551 \quad$ (2017), https://doi.org/10.3109/10837450.2016.1163385

15 S. Y. Karavana, S. Rencber, Z. Ay Şenyiğit and E. Baloglu, Pharmacol. Pharm., 3, 417 (2012), https://doi.org/10.4236/pp.2012.34056

16 M. Ozyazici, M. Firlak, S. Tuncay Tanriverdi, S. Rencber, S. Y. Karavana et al., Am. J. Drug Deliv. Therap., 2, 78 (2015), http://www.imedpub.com/abstract/bioadhesive-geland-hydrogel-systems-forrnbuccal-delivery-ofketoprofen-preparationrnand-in-vitro-evaluationstudies-11053.html

17 S. Y. Karavana, E. Homan Gokçe, S. Rencber, S. Ozbal, C. Pekcetin et al., Int. J. Nanom., 7, 5693 (2012), https://doi.org/10.2147/IJN.S36883

18 D. S. Jones, A. D. Woolfson and J. J. Dkokic, Appl. Polym. Sci., $\quad$ 61, $2229 \quad$ (1996), https://doi.org/10.1002/(SICI)10974628(19960919)61:12<2229::AID-APP24>3.0.CO;2-0 19 S. Rodino, M. Butu, C. Negoescu, A. Caunii, R. T. Cristina et al., Digest. J. Nanomater. Biostructr., 9, 1215

(2014), http://www.chalcogen.ro/1215_Butnariu.pdf

20 S. Ramachandran, S. Chen and F. J. Etzler, Drug Dev. Ind. Pharm., 25, $153 \quad$ (1999), https://doi.org/10.1081/DDC-100102155

21 A. Cojocariu, L. Profire, C. N. Cheaburu, A. M. Oprea and C. Vasile, E-Polymers, 30, 1 (2011), https://doi.org/10.1515/epoly.2011.11.1.334

22 C. N. Cheaburu-Yilmaz, O. Yilmaz and C. Vasile, Adv. Struct. Mater., 74, $341 \quad$ (2015), https://www.springerprofessional.de/en/polysaccharide -containing-gels-for-pharmaceuticalapplications/15995476

A. Cojocariu, L. Porfire, C. Cheaburu and C. Vasile, Cellulose Chem. Technol., 46, 35 (2012), http://www.cellulosechemtechnol.ro/pdf/CCT12(2012)/p.35-43.pdf

24 E. Parparita, C. N. Cheaburu and C. Vasile, Cellulose Chem. Technol., 46, 571 (2012), http://www.cellulosechemtechnol.ro/pdf/CCT46,9-

10(2012)/p.571-581.pdf

25 C. N. Cheaburu, O. N. Ciocoiu, G. Staikos and C. Vasile, J. Appl. Polym. Sci., 127, 3340 (2013), https://doi.org/10.1002/app.37789

26 T. G. Mezger, "The Rheology Handbook", $2^{\text {nd }}$ ed., Vincentz Network GmbH \& Co. KG, Hannover, Germany, 2006 , https://www.semanticscholar.org/paper/The-rheologyhandbook-\%3A-for-users-of-rotational-andMezger/d5cf75a5e4dd074b1bf54b905f8c609b6e806e2

27 C. N. Cheaburu-Yilmaz, S. Tuncay Tanriverdi, O. Ozer and C. Vasile, in "Handbook on Polymer Gels", edited by V. K. Thakur and M. K. Thakur, Springer Nature, Switzerland AG, 2018, pp. 231-278, https://www.springer.com/gp/book/9789811060854

28 A. Kurrey, P. K. Suresh and M. R. Singh, Chron. Young Sci., 5, 1 (2014), https://doi.org/10.4103/2229. 5186.129327

29 N. M. Harish, P. Prabhu, R. N. Charyulu, M. A. Gulzar and E. V. S. Subrahmanyam, Indian J. Pharm. Sci., 71, 421 (2009), https://doi.org/10.4103/0250474X.57291

30 C. N. Cheaburu-Yilmaz, R. P. Dumitriu, M. T. Nistor, C. Lupusoru, M. I. Popa et al., British J. Pharm. Res., 6, $228 \quad$ (2015), https://doi.org/10.9734/BJPR/2015/16525

31 Y. C. Marcano and M. A. Sabino, Cellulose Chem. Technol., $\quad 52, \quad 559 \quad$ (2018), http://www.cellulosechemtechnol.ro/pdf/CCT78(2018)/p.559-567.pdf

32 M. Popa, B. C. Ciobanu, L. Ochiuz, J. Desbrieres, C. S. Stan et al., Cellulose Chem. Technol., 52, 353 (2018),

http://www.cellulosechemtechnol.ro/pdf/CCT56(2018)/p.\%20353-370.pdf 